\title{
Using adjoint sensitivity as a local structure function in variational data assimilation
}

\author{
G. Hello ${ }^{1}$ and F. Bouttier ${ }^{2}$ \\ ${ }^{1}$ Centre National de Recherche de la Météorologie, Météo-France, Toulouse, France \\ ${ }^{2}$ European Centre for Medium range Weather Forecasts, Reading, UK
}

Received: 18 September 2000 - Revised: 24 May 2001 - Accepted: 2 July 2001

\begin{abstract}
One approach recently proposed in order to improve the forecast of weather events, such as cyclogenesis, is to increase the number of observations in areas depending on the flow configuration. These areas are obtained using, for example, the sensitivity to initial conditions of a selected predicted cyclone. An alternative or complementary way is proposed here. The idea is to employ such an adjoint sensitivity field as a local structure function within variational data assimilation, 3D-Var in this instance. Away from the sensitive area, observation increments project on the initial fields with the usual climatological (or weakly flow-dependent, in the case of 4D-Var) structure functions. Within the sensitive area, the gradient fields are projected using all the available data in the zone, conventional or extra, if any. The formulation of the technique is given and the approach is further explained by using a simple 1D scheme. The technique is implemented in the ARPEGE/IFS code and applied to 11 FASTEX (Fronts and Atlantic Storm-Track Experiment) cyclone cases, together with the targeted observations performed at the time of the campaign. The new approach is shown to allow for the desired stronger impact of the available observations and to systematically improve the forecasts of the FASTEX cyclones, unlike the standard 3D-Var.
\end{abstract}

\section{Introduction}

Short range numerical forecasting of rapidly evolving meteorological events, such as cyclogeneses (with less than a $48 \mathrm{~h}$ range), remains a challenge despite the use of highly sophisticated numerical models and data assimilation procedures. These events are not very frequent but they can cause severe damages. This is why it is most important to improve the forecasts in these cases. The FASTEX (Fronts and Atlantic Storm-Track Experiment, Joly et al., 1999) project provides the background motivation for the present study: one of its

Correspondence to: G. Hello (gwenaelle.hello@meteo.fr) objectives is to improve the forecast of the North Atlantic cyclones hitting Europe.

One solution developed recently is the concept of "targeting" (see Emanuel et al., 1995, for a first reference to adaptive observations). The approach is to increase the number of observations in locations determined by the dynamics of the flow of the day. The extra data collected is assimilated using standard data assimilation techniques: the information used to locate the extra data in space is forgotten. The different techniques used so far to determine the locations of the extra data are of two kinds. One uses the adjoint tool (Rabier et al., 1996) and the other one uses ensemble techniques (Bishop and Toth, 1999). Some real time implementations of these ideas are available for reference (Bergot, 1999; Szunyogh et al., 1999). The results show overall a positive impact of these dedicated observations on the forecasts and the authors point out the fact that degradation of the forecasts could also happen. To go a step further, Bergot (2000) shows that this gain is, nevertheless, highly dependent on the data assimilation procedure used on these observations. This suggests to improve in the assimilation the use of these specific types of observations that are located in crucial areas for the forecast quality. One idea which is the baseline of the present work is to introduce the predictability information previously used to determine the locations where the number of observations should increase within the framework of variational data assimilation.

On selected cases, Hello et al. (2000) have shown that correcting the analysis with such information does bring a significant improvement in the forecast. The correcting perturbation to the analysis state is derived from the sensitivities to the initial conditions of a forecast cyclone. It is then added to the current analysis and scaled by the available observations inside the sensitive areas. This is a very crude but efficient way to address the previous need. Nevertheless, the procedure stands outside of the assimilation framework. As a result, the statistical significance of the final initial state is not known. The present study tries to keep the same spirit but within the framework of data assimilation. It shows how an 
adjoint sensitivity field that computes the $\sim 48 \mathrm{~h}$ sensitivity to initial conditions of a weather system of interest (here, a predicted cyclone) can be introduced in the background error matrix B. Away from the sensitive area, the usual climatological structure functions are used to project the observed data on the initial fields. Within the sensitive area, a flow dependent structure function is employed. The article shows how this behaviour can be formulated in the framework of the socalled Simplified Kalman Filter (Fisher, 1998) in Sect. 2. The approach is further explained within a simple 1D variational problem in Sect. 3. Finally, it is applied in Sect. 4 to a series of 11 FASTEX cyclone cases, the forecast of which is known to be difficult to improve. Yet, this new approach allows for a better forecast. This new technique may be thought of as providing the same benefit as a 4D-Var assimilating observation at a time far away from the nominal analysis time. However, it does so at a fraction of the cost and only locally: in that sense, this approach is a targeted data assimilation scheme focused on a given weather system.

\section{Formulation}

In order to insert a specific direction for projecting observation increments inside an assimilation procedure, it is necessary to carry out the two following steps. The first one is to provide the direction. This is done in the spirit of targeting (see Snyder (1996) for a first application of the adaptive observations concept to the FASTEX (Joly et al., 1999) field experiment). The idea is as follows. Given a meteorological system for which the numerical short range forecast has to be improved, it is possible to compute one or more directions in which analysis errors are likely to be rapidly amplified in the forecast, even if they are small.

The second step is to introduce this sensitive direction within an assimilation procedure. This study is done within a 3D-Var framework (Courtier et al., 1998). The modification is done on the background term of the 3D-Var and more precisely, to the background error covariance matrix $\mathbf{B}$. The idea is to replace the climatological values of the variance of the background errors by large ones for the part of the control variable that projects onto this sensitive direction. In the limit case, an infinite value could be set, meaning that no confidence is given to the background field in this sensitive direction. It was convenient to formulate the problem in the framework of the so-called simplified Kalman filter (hereafter denoted SKF), developed and implemented at the European Centre (ECMWF) (Fisher, 1998).

The modification is applied to the $J_{b}$ term of the assimilation cost function of the 3D-Var that measures the distance to the background field. This is done directly on the covariance matrix of the background error which leads to splitting $J_{b}$ into three different parts:

$$
J_{b}(\chi)=\underbrace{\frac{1}{2} \chi_{S}{ }^{T} \mathbf{B}_{\chi}{ }^{-1} \chi_{S}}_{1}+\underbrace{\chi_{S}{ }^{T} \mathbf{B}_{\boldsymbol{\chi}}{ }^{-1} \chi_{\bar{S}}}_{2}+\underbrace{\frac{1}{2} \chi_{\bar{S}}{ }^{T} \chi_{\bar{S}}}_{3},
$$

where $\mathbf{B}_{\chi}$ is the background error matrix for the control variable $\chi$, which is related to the state variable $\boldsymbol{x}$ by the usual change of variable $\mathbf{L}, \chi=\mathbf{L}^{-1} \delta \boldsymbol{x}=\mathbf{B}_{\bar{S}}^{-1 / 2} \delta \boldsymbol{x}$ in the $3 \mathrm{D}$ and $4 \mathrm{D}$ variational analysis of the ARPEGE/IFS model (Bouttier et al., 1997).

For $\boldsymbol{x} \in S, \mathbf{B}_{\chi}^{-1}=\mathbf{L}^{T} \mathbf{B}_{S}^{-1} \mathbf{L}$ and for $\boldsymbol{x} \in \bar{S}$, the third term is equal to:

$$
\frac{1}{2} \delta \boldsymbol{x}_{\bar{S}}^{T} \mathbf{B}_{\bar{S}}^{-1} \delta \boldsymbol{x}_{\bar{S}}=\frac{1}{2}\left(\mathbf{B}_{\bar{S}}^{-1 / 2} \delta \boldsymbol{x}_{\overline{\mathbf{S}}}\right)^{T}\left(\mathbf{B}_{\bar{S}}^{-1 / 2} \delta \boldsymbol{x}_{\bar{S}}\right)=\frac{1}{2} \chi_{\bar{S}}^{T} \chi_{\bar{S}} .
$$

Term 1 represents the part of the state variable that projects onto the subspace $S$, defined by the sensitive direction hereafter denoted as $\boldsymbol{y}_{1}$, which is a gradient of a function of the forecast cyclone with respect to its initial conditions, as described in Hello et al. (2000): the function is calculated using the forecast and it is not in retrospect. Term 2 concerns the cross products, and term 3 concerns the part of the state variable that projects onto the orthogonal of the subspace $S, \bar{S}$. The $J_{b}$ term here is of the same form as in the usual 3D variational framework.

The inverse of the covariance matrix of the background error $\mathbf{B}_{\chi}^{-1}$ is assumed to have the following shape:

$\mathbf{X}\left(\begin{array}{ccc}f_{1} & \cdots & f_{n} \\ \vdots & \mathbf{G} \\ f_{n} & \end{array}\right) \mathbf{X}^{T}$

where $n$ is the dimension of the control variable $\chi$. The matrix $\mathbf{X}$ is a Householder matrix (see Appendix) applied to the vector $\mathbf{L}^{-1} \boldsymbol{y}_{1}$. The application of the matrix $\mathbf{X}$ to the vector $\mathbf{L}^{-1} \boldsymbol{y}_{1}$ is a vector that has all its components equal to zero, except the first one. It acts as a rotation and thus: $\mathbf{X X}^{T}=\mathbf{I}$, where $\mathbf{I}$ is the identity matrix. The scalars $\left(f_{i}\right)$ are the components of the first row and the first column of the matrix between $\mathbf{X}$ and $\mathbf{X}^{T}$. The matrix $\mathbf{G}$ is a sub-matrix of rank $n-1$.

It can be shown that:

$$
\left(\begin{array}{c}
f_{1} \\
\vdots \\
f_{n}
\end{array}\right)=\frac{1}{\sigma_{1}^{2}\left\|\mathbf{L}^{-1} \boldsymbol{y}_{1}\right\|} \mathbf{X}^{T} \mathbf{L}^{T} \mathbf{L} \boldsymbol{y}_{1}
$$

$\mathbf{G}=\mathbf{I}_{n-1}$

The parameter $\sigma_{1}^{2}$ is the constraint imposed on the amplitude of the variance in the sensitive direction $\boldsymbol{y}_{1}$ within the background error matrix.The matrix $\mathbf{I}_{n-1}$ is the identity matrix of rank $n-1$.

The modification then takes the form of a change in the variable on top of the one currently used $(\mathbf{L})$ in the variational framework. The new change in the variable is noted as $\tilde{\mathbf{L}}$ :

$$
\delta \boldsymbol{x}=\mathbf{L} \chi=\mathbf{L} \tilde{\mathbf{L}} \tilde{\chi}=\mathbf{L X} \tilde{\mathbf{I}} \tilde{\chi},
$$


where $\delta \boldsymbol{x}$ is the increment of the state variable to the background field. The $\tilde{\mathbf{I}}$ matrix takes the following form:

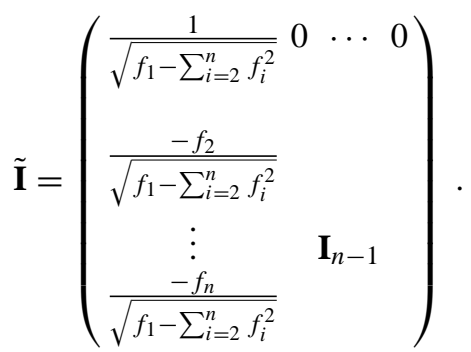

The functional $J$, including both the background and the observation contributions, can be rewritten in terms of $\tilde{\chi}$ as:

$$
\begin{aligned}
J(\tilde{\chi})= & \frac{1}{2} \tilde{\chi}^{T} \tilde{\chi} \\
& +\frac{1}{2}(\mathbf{H L} \tilde{\mathbf{L}} \tilde{\chi}-\boldsymbol{d})^{T} \mathbf{R}^{-1}(\mathbf{H L} \tilde{\mathbf{L}} \tilde{\chi}-\boldsymbol{d}) .
\end{aligned}
$$

$\mathbf{R}$ is the covariance matrix of observation errors, $\boldsymbol{d}=\boldsymbol{y}-$ $H \boldsymbol{x}_{b}$ is the innovation vector, $\boldsymbol{y}$ is the vector of observation values, $H$ is the observation operator and $\mathbf{H}$ is a linear approximation of it. The vector $\boldsymbol{x}_{b}$ is the background state variable. The first term on the right-hand side of the Eq. (7) is the new form of Eq. (1).

A proper state variable $\boldsymbol{x}$ can be recovered from:

$$
\begin{aligned}
& \delta \boldsymbol{x}=\mathbf{L} \tilde{\mathbf{L}} \tilde{\boldsymbol{\chi}} \\
& \quad=\mathbf{L} \tilde{\mathbf{L}}(\mathbf{L} \tilde{\mathbf{L}})^{T} \mathbf{H}^{T}\left[\mathbf{R}+\mathbf{H L} \tilde{\mathbf{L}}(\mathbf{H L L} \tilde{\mathbf{L}})^{T}\right]^{-1} \boldsymbol{d} .
\end{aligned}
$$

It can be seen that the standard 3D-Var procedure is recovered by replacing $\mathbf{X} \tilde{\mathbf{I}}=\tilde{\mathbf{L}}$ by the identity matrix in the equations.

\section{One-dimensional variational application}

To illustrate the behaviour of the method, a toy model is now used. It is the one built by Desroziers and Ivanov (2000) in order to assess the adaptive tuning of information errors in a variational assimilation. The geometry considered in this toy model is a circular domain that can be seen as an Earth parallel on a sphere. The perimeter is set to $P=2 \pi a$, where $a$ is the Earth's radius. The field $x$ at the coordinate location $s$ can then be expressed as Fourier series:

$x(s)=\frac{1}{M} \sum_{k=-K}^{k=K} \widehat{x_{k}} \exp \left(\frac{2 \pi i k s}{P}\right)$.

The truncation (maximum wave number) used here to estimate the real $x$ is $K=100$, which leads to $M=201$ spectral coefficients $\widehat{x_{k}}$. The matrix $\mathbf{B}$ is defined in the physical space by an isotropic Gaussian correlation function with a lengthscale of $300 \mathrm{~km}$. The observation operator $H$ is considered to be linear. The sensitive direction $\boldsymbol{y}_{1}$ is fixed as:

$$
y_{1}=\exp \left\{-\frac{1}{2}\left(\frac{s-P / 2}{L_{G}}\right)^{2}\right\} \cos \left\{\frac{4(s-P / 2)}{L_{G}}\right\} \text {. }
$$
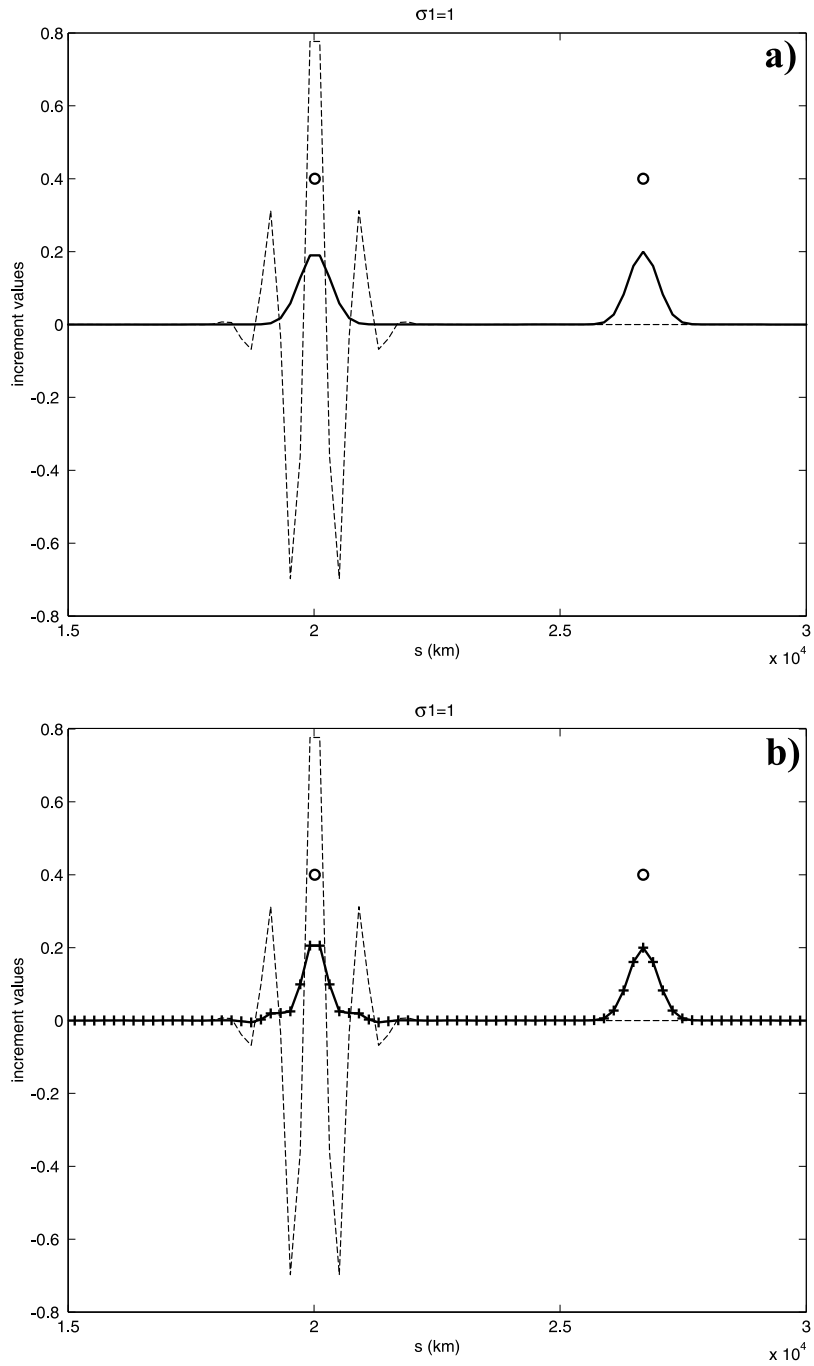

Fig. 1. The circles are the observations. The sensitive direction $y_{1}$ is given by the thin dashed line, The value $\sigma_{1}$ is set to 1 . (a) Increment resulting from a standard 3D-Var (thick solid line). (b) Increment given by the modified $J_{b}$ term (crosses connected with the thick solid line).

This is done in order to mimic the usual gradient shape that can be observed, for example, in a targeted framework (series of positive and negative lobes). The length-scale $L_{G} / 4$, $\left(L_{G}\right.$ is $600 \mathrm{~km}$ ) chosen for $\boldsymbol{y}_{1}$ is half the scale set in the definition of the matrix $\mathbf{B}$. This ratio mimics what a real experiment shows. The characteristic model state is given by the background field which is set to zero for the sake of simplicity. In a first part, two observations are considered (Fig. 1 and Fig. 2). The first one is in a part of the circular domain where the sensitive pattern $\boldsymbol{y}_{1}$ is not zero. The second one is in a part of the domain where $\boldsymbol{y}_{1}$ is zero. Considering these two observations enables one to compare the behaviour of the increment of the analysis for the part that belongs to the sensitive direction and for the one that does not. Thanks to the simplicity of this framework, it is possible to compute explicitly the increment of the analysis by using the expression of $\delta \boldsymbol{x}$ in Eq. (8). 

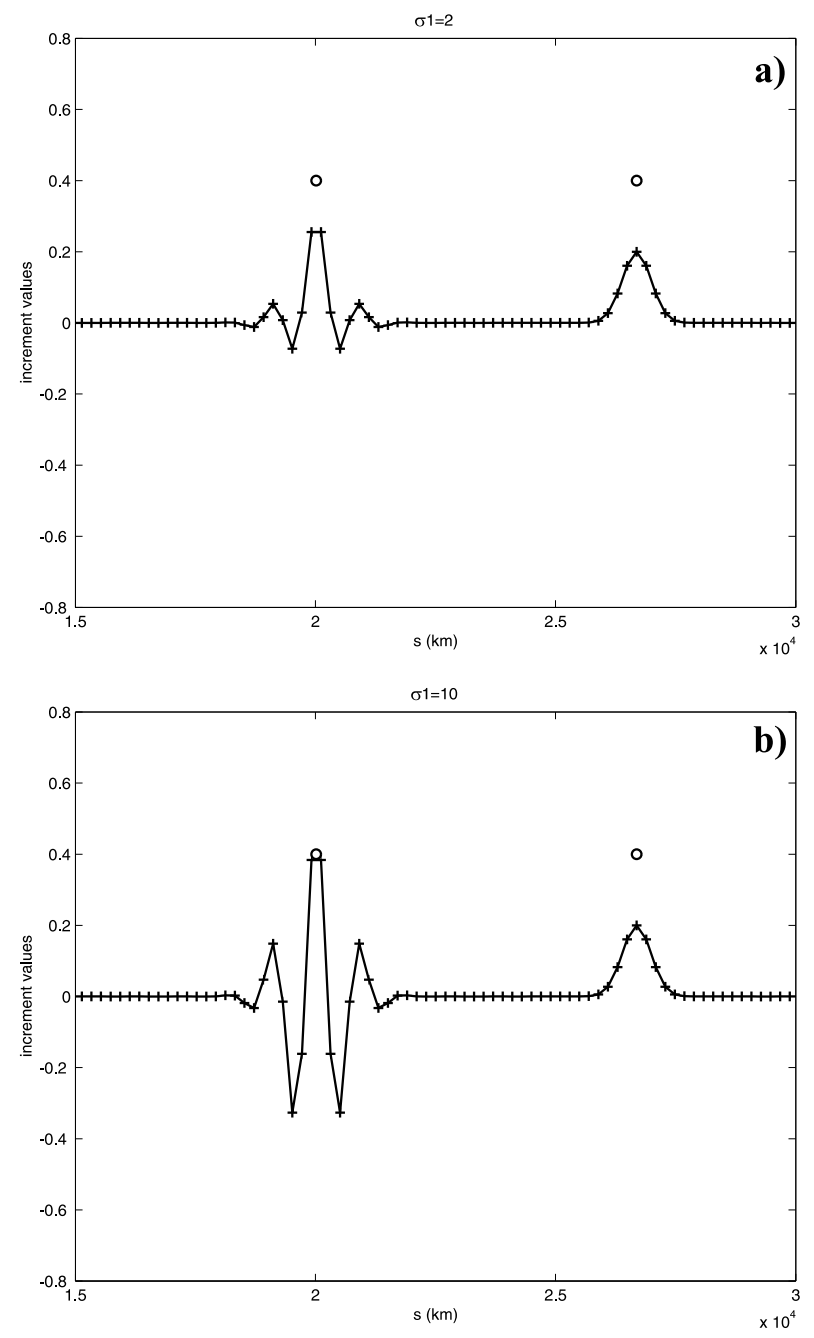

Fig. 2. The circles are the observations. The thick solid lines with crosses are the increments given by the modified $J_{b}$ term. (a) $\sigma_{1}$ is set to 2. (b) $\sigma_{1}$ is set to 10 .

At the location of the two observations, Fig. 1, panel (a) shows the increments obtained with the standard 3D-Var. Their amplitudes are half the value of the observations at their locations. This is due to the fact that $\sigma_{o}=\sigma_{b}=1$ in these experiments. The comparison of panel (a) and panel (b) in Fig. 1 shows that for the observation taken where $\boldsymbol{y}_{1}=0$, increments from the standard and from the new method are the same. This occurs because the modification has, by definition, no effect where $\boldsymbol{y}_{1}=0$. This is implemented by matrix $\mathbf{X}$ : in such areas, $\mathbf{X} \tilde{\mathbf{I}}=\mathbf{I}$. For the other observation, located where $\boldsymbol{y}_{1} \neq 0$ (i.e. in the sensitive area), the increment coming from the new procedure is nearly the same as the one from the standard 3D-Var. This occurs because in Fig. 1, the value of $\sigma_{1}$ is set to one, and in this specific case, the matrix $\mathbf{X} \tilde{\mathbf{I}}$ is not very different from the identity matrix. In Fig. 2, different values of the parameter $\sigma_{1}$ are used. It shows that the shape of the increment is closing in on that of the $\boldsymbol{y}_{1}$ direction for the first observation, while it remains unaffected for the other observation. Near the first observation,
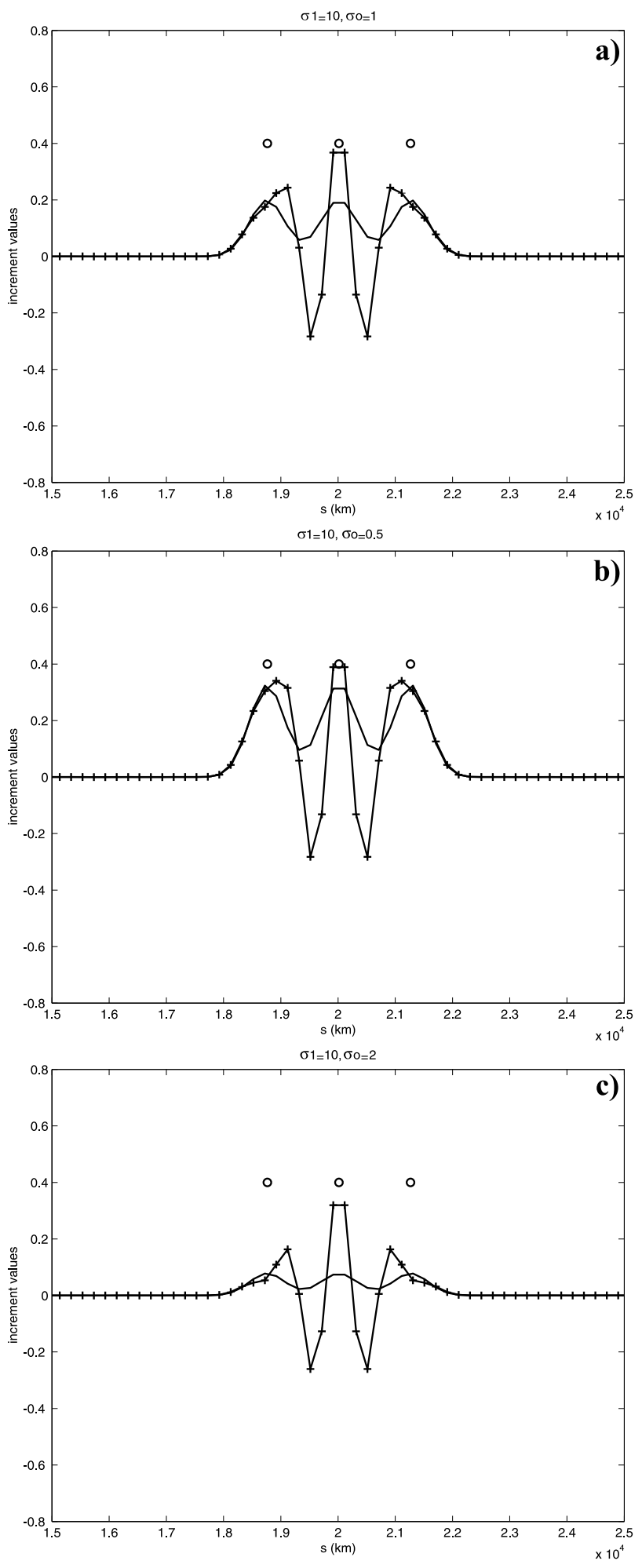

Fig. 3. The circles are the observations. $\sigma_{1}$ is set to 10 . The solid lines are the increments resulting from a standard 3D-Var. Increments resulting from the modified 3D-Var are plotted with crosses connected with solid lines. (a) $\sigma_{o}$ is set to 1 . (b) $\sigma_{o}$ is set to 0.5. (c) $\sigma_{o}$ is set to 2 .

a high value of $\sigma_{1}$ leads to a perfect fit of both the observation and the shape of the sensitivity function $y_{1}$ (panel (b) 

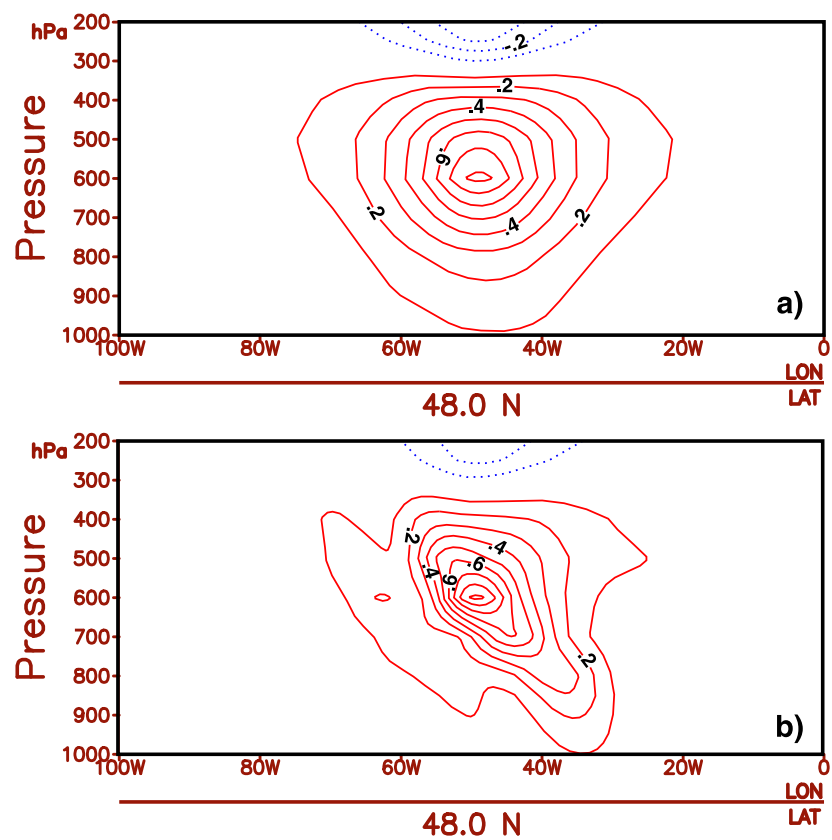

Fig. 4. Vertical cross section of an analysis increment of a temperature field for a single temperature observation experiment (contour interval 0, $1 \mathrm{~K}$ ). (a) standard 3D-Var. (b) modified 3D-Var. $\sigma_{1}$ is set to $10^{2}$.

on Fig. 2). This fit to the observation value for large values of the parameter $\sigma_{1}$ seems perfect in the sensitive area due to the crude definition of this model: $\sigma_{o}$ is set to one and there is only one observation located exactly where $\boldsymbol{y}_{1}$ is the largest. In reality, the adjustment is more complex. It depends on several parameters such as the values of the $\sigma_{o}$ used to set the matrix $\mathbf{R}$ of observation errors as one expected, the number of observations and also their locations with respect to the amplitude of $\boldsymbol{y}_{1}$. Figure 3 is focusing on the area where $\boldsymbol{y}_{1} \neq 0$. The increments correcting the background are computed with large $\sigma_{1}$. Panel (a) of Fig. 3 shows the same lines, fields, and structures as in panel (b) of Fig. $2\left(\sigma_{1}=10\right.$, $\sigma_{o}=1$ ), but using three observations. First, it can be seen that the adjustment for the second observation is no longer equal to the observation value. We can also see in panel (a) of Fig. 3 that the amplitude of the increment changes as the locations of the observations are modified. The increment due to the second observation is larger than the two others because this observation is located in a place where the amplitude of $\boldsymbol{y}_{1}$ is larger. In panel (b) of Fig. 3, the value of $\sigma_{o}$ is set to 0.5, meaning that the observations are assumed to have less errors. We can see now that the increments fit the observations values better. This is also the case for the standard 3D-Var increments. On the contrary, panel (c) of Fig. 3 shows observations with more errors ( $\sigma_{o}$ is set to 2$)$. The increments are now not as close to the observations. Nevertheless, the shape of the increments, due to the modification of the $J_{b}$ term, has always followed the shape of $\boldsymbol{y}_{1}$.

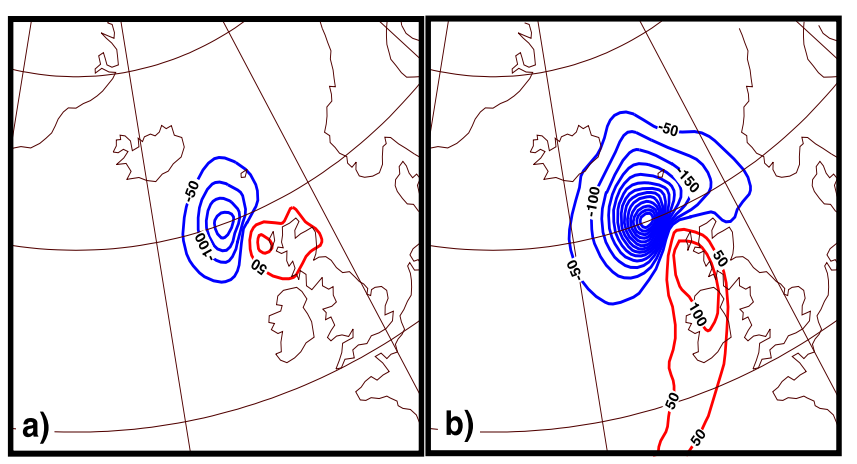

Fig. 5. Impact of the assimilation of one temperature observation on the forecast. Differences in the mean sea level pressure field between the 36-h forecast coming from an analysis, and the 36-h forecast coming from the background field (contour interval $50 \mathrm{~Pa}$ ). (a) The analysis is a 3D-Var. (b) The analysis is a modified 3D-Var. $\sigma_{1}$ is set to $10^{2}$.

\section{Application to the targeted FASTEX data}

\subsection{Single observation experiments}

The analysis increment resulting from a single observation experiment gives access to the implicit effective structure functions of the analysis. Such experiments are performed in the framework of the Arpege 3D-Var, in order to compare the structure functions used in the standard 3D-Var system and the ones used in the modified 3D-Var system. Arpege is a global spectral model (Courtier et al., 1991) with a variable mesh, providing a very high resolution near the pole of interest. The variational assimilation scheme is based on an incremental approach (Thépaut et al., 1998). For the experiment, a T199 resolution is used with a stretching factor of 3.5 for the trajectory, and a T63 with no stretching used for the increments.

The observation chosen is coming from a so-called FASTEX "targeted" flight. The reader is referred to Joly et al. (1999) for a summary of the FASTEX field phase. This flight is part of IOP17 (Intense Observing Period) (Cammas et al., 1999), with a verifying time at 12:00 UTC on 19 February and a target time at 00:00 UTC on 18 February. The observation is chosen because it is located inside a maximum of sensitivity to initial conditions at a target time of the forecast low at the verifying time.

Figure 4 shows the comparison of the two different analysis increments. For the 3D-Var analysis (panel (a) in Fig. 4), the structure function is isotropic. It is no longer the case with the modified 3D-Var analysis (panel (b) in Fig. 4), as the structure function takes on the shape of the gradient field used as the sensitive direction. The increment thus adopts the vertical baroclinic tilt that is often noticed in linear modes and structures related to mid-latitude cyclogenesis. This is precisely what the technique is meant to do. The effect of the two analysis procedures on the 36-h forecast at time 12:00 UTC on 19 February is shown in Fig. 5: the effect of 

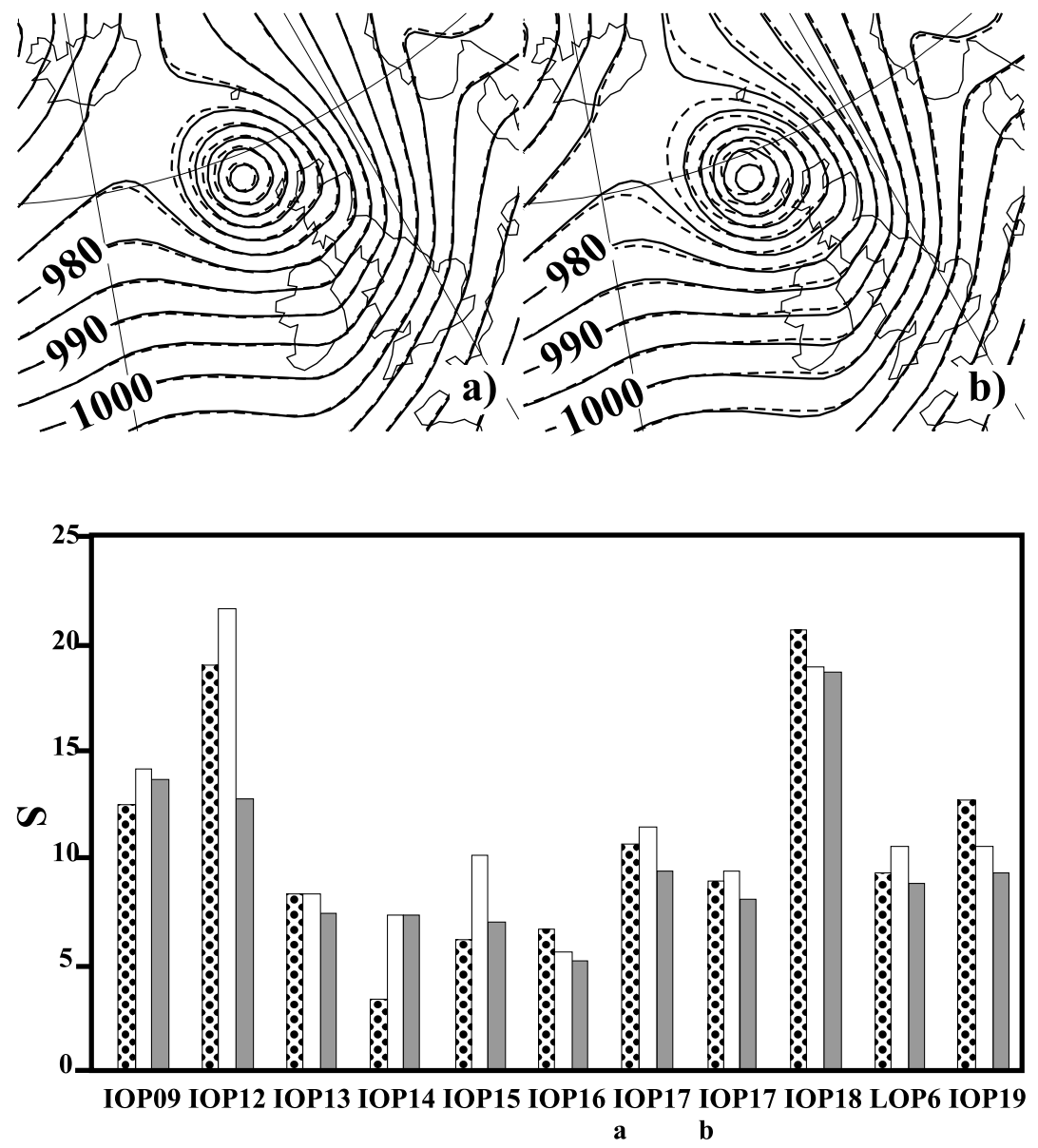

Fig. 6. Impact of the assimilation of one observation on the 36-h forecast mean sea level pressure field for IOP17b. The contour is $5 \mathrm{hPa}$. Solid lines: the no analysis forecast. (a) dashed lines: the forecast coming from the standard 3DVar analysis. (b) dashed lines: the forecast coming from the modified 3D-Var analysis.
Fig. 7. Limited area $S$ score of the forecasts for the 11 targeted flights of FASTEX. Dotted bars: the no analysis forecast. White bars: forecast made with the standard 3D-Var analysis. Dark grey bars: forecast made with the modified 3D-Var analysis. the modified 3D-Var is clearly larger. The impact of the assimilation of one observation on the forecast field itself is shown in Fig. 6. We see from this figure that the effect of the assimilation of a single observation is to move the low of IOP17b towards the northwest. This displacement is amplified with the modified assimilation (dashed lines in Fig. 6, panel (b) to be compared with the ones of panel (a)). The new forecast location of this low is in agreement with the actual location of the low of IOP17b: the reference 4D-Var re-analysis is given at $59 \mathrm{~N}$ and $12,7 \mathrm{~W}$ for the low location. In this case, the standard 3D-Var brings an improvement, and the modified 3D-Var improves the location even more. The impact of one observation inside a maximum of sensitivity at 00:00 UTC on 18 February is $2 \mathrm{hPa}$ on the 36 -h forecast, at 12:00 UTC on 19 February for the standard 3D-Var, and $6.5 \mathrm{hPa}$ for the modified 3D-Var. It can be pointed out that although the value of the increment at the observation point does not differ too much in the two cases (less than $0.1 \mathrm{~K}$ ), the impact on the forecast can be three times larger only due to the modification of the shape of the structure function. The same kind of results where shown, for example, by Thépaut et al. (1996) with the comparison between single observation experiments with a 4D-Var and with a 3D-Var assimilation. As is the case for the modified 3D-Var studied here, the 4DVar uses dynamical structure functions, but it defines them implicitly.

\subsection{The targeted flights experiments}

The experiments are now performed with all the observations coming from the targeted flights that were performed during the FASTEX campaign. An evaluation of the 20 targeted flights of FASTEX can be found in Bergot (1999). Eleven flights are used here covering the following IOPs: 9, 12 to 19 and LOP6 (Lesser Observations Period). The sensitive direction $\boldsymbol{y}_{1}$ is computed with the forecast trajectory of the $11 \mathrm{cy}-$ clones following the definition of the target and verification times, as described in Bergot (1999). The verification areas are centered on the location of the cyclones at the time of the verification. The value of $\sigma_{1}$ is set to infinity: no confidence is given to the background field within the sensitive area.

\subsubsection{Score of the different forecasts with respect to the} available observations inside the verification areas

The score $S$ of the different forecasts at the verification time is computed with respect to the observations by using the $J o$ term of the analysis procedure restricted to the verification area:

$S=\frac{1}{N_{\mathrm{obs}}}\left(H\left(\boldsymbol{x}_{f}\right)-\boldsymbol{y}\right)^{T} \mathbf{R}^{-1}\left(H\left(\boldsymbol{x}_{f}\right)-\boldsymbol{y}\right)$, 


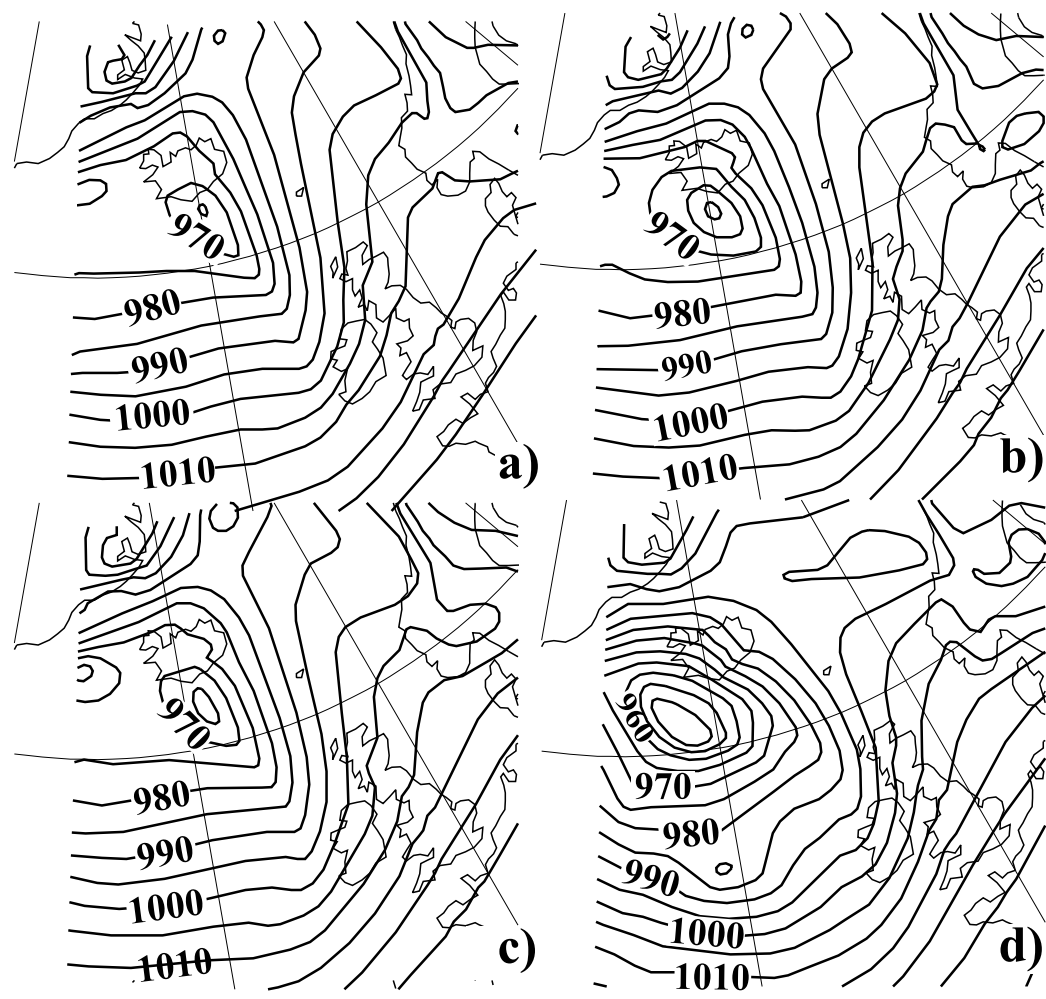

Fig. 8. IOP12. Mean sea level pressure field (contour interval $5 \mathrm{hPa}$ ). 36-hour forecasts from different initial conditions: (a) 3D-Var analysis, (b) modified 3D-Var analysis, (c) background. Reference analysis of IOP12: (d) FASTEX 4D-Var re-analysis. where $N_{\text {obs }}$ is the number of available observations inside the verification area. The vector $\boldsymbol{y}$ is the list of observed values, the vector $\boldsymbol{x}_{f}$ is the forecast coming from a 3D-Var procedure or a modified 3D-Var procedure. $\mathbf{R}$ is the covariance matrix of observational errors and $H$ is the observational operator that allows one to compute model values at observations locations.

The results are shown in Fig. 7. First, it is important to notice that it is difficult for the standard 3D-Var assimilation procedure to improve the score of the background. As an average, the score of the forecasts issued from the standard 3D-Var with respect to the observations is $8 \%$ worse than the ones derived from the background (with no assimilation). For the 11 flights, the score of the forecast with a 3D-Var is better than the one with no analysis (background field) but only for 3 times: IOP16, 18 and 19. First, we can see here that the FASTEX cases were usually well predicted during the two months and that it is difficult to see an impact on the forecasts that already have a good quality. Added to this, we can also see there that a 3D-Var assimilation procedure is not ideal for using targeted observations, as was shown in Bergot (2000). This is less true for the forecasts coming from a modified 3D-Var. On the contrary, except for IOP9, 14 and 15, the score of the forecasts coming from a modified 3D-Var is better than the forecasts coming from the background. As an average, the score of the forecasts derived from the modifed 3D-Var is a little more than $10 \%$ better than the ones derived from the background. It is also noticeable that for all the cases, the forecasts coming from the modified 3D-Var analysis perform better than the ones coming from the non-modified 3D-Var analysis. Figure 8 shows the forecast fields for IOP12. The reference (Fig. 8, panel d) is the 4D-Var FASTEX re-analysis (Desroziers et al., 1999). It is a very good reference field as it includes all the extra observations (special soundings from ships, land and special flights) that have been performed during the FASTEX field phase. We can see in Fig. 8 that starting from the background (Fig. 8, panel c), the standard 3D-Var procedure is worsening the forecast (Fig. 8, panel a), while the modified 3D-Var is improving it. Figure 8, panel (b) is closer to the reference panel (d).

\subsubsection{Score of the different forecasts with respect to the FASTEX 4D-Var re-analysis inside the verification areas}

We now used the 4D-Var re-analysis of the FASTEX period (Desroziers et al., 1999) as a reference in order to compute the scores of the different forecasts. By doing so, we avoid the problem that could exist with the $S$ score when too few observations occur to be able to make a comparison to the forecasts. Although the unusually large amount of data available about the FASTEX cyclone cases in the vicinity of Europe prevents this risk, the following score allows for a fieldto-field comparison. The scores of the forecasts are computed as an rms improvement. The rms improvement is defined as follows:

$\operatorname{rms}$ improvement $(\%)=100\left[1-\frac{\operatorname{rms}\left(f_{c}\left(X_{a}\right)\right)}{\operatorname{rms}\left(f_{c}\left(X_{b c k}\right)\right)}\right]$, 


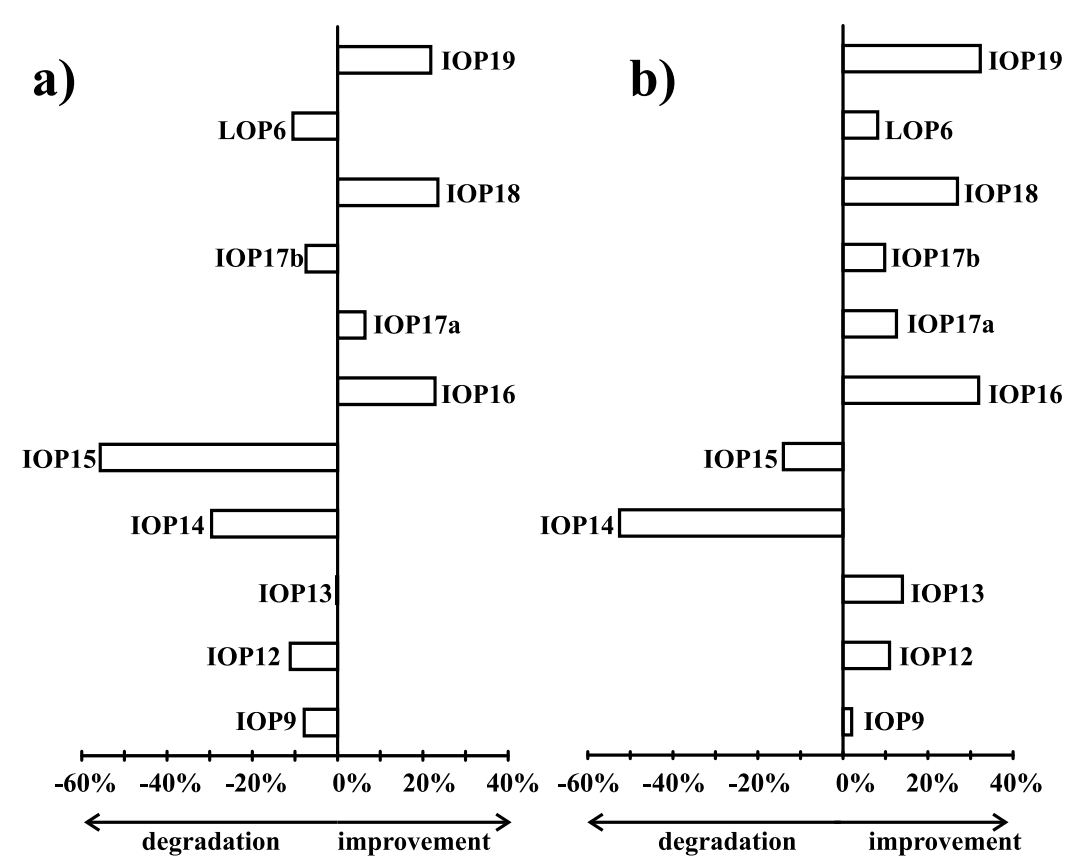

Fig. 9. Rms improvement with respect to the background of the mean sea level forecast pressure field inside the verification area derived from different analysis for the 11 targeted flights of FASTEX. The reference is the 4D-Var FASTEX re-analysis. (a) 3D-Var analysis, (b) modified 3D-Var analysis. where $X_{a}$ is the analysis (standard 3D-Var or modified 3DVar) and $f_{c}$ is the forecast mean sea level pressure coming from this analysis. The field $X_{b c k}$ is the background (no assimilation) and $f_{c}\left(X_{b c k}\right)$ is the forecast mean sea level pressure coming from the background field. The computation of the rms is done over the verification area and uses the 4D-Var re-analysis as reference:

$\operatorname{rms}(f c(X))=\sqrt{\frac{\sum_{i \in \Omega}\left(f_{c}\left(X_{i}\right)-X_{i}^{r e f}\right)^{2} \cos \varphi_{i}}{\sum_{i \in \Omega} \cos \varphi_{i}}}$,

where $\Omega$ is the verification area, $\varphi$ is the latitude of the grid point $i$ and $X^{r e f}$ is the 4D-Var re-analysis used as a reference. This score tells us the impact of the analysis procedure on the quality of the forecast. The results are shown in Fig. 9. In panel (a) of this figure, we see the improvement or the degradation on the forecasts implied by the standard 3D-Var procedure to be compared with the forecast without analysis. Out of the 11 cases, the forecast improves only 4 times (IOP16, IOP17a, IOP18 and IOP19). We find here again the same result as for the $S$ score, except for IOP17a. On average, the standard 3D-Var procedure is degrading the non-analysis forecast by $4 \%$. In panel (b) of Fig. 9, the improvement or the degradation of the forecast resulting from the modified 3D-Var analysis is shown. The results are the opposite: nearly all the forecasts are improved, with only two exceptions: IOP14 and IOP15. The same results as for the $S$ score against the observations are found, except for IOP9, which now reveals a slight improvement on this parameter. On average, the modified 3D-Var procedure is improving the non-analysis forecast by $8.7 \%$.

It can be concluded from these two scores that the modified procedure performs better than the standard 3D-Var with the targeted observations. This could be a way to take into account this type of observation more properly at only small extra cost.

\section{Conclusions}

A local, "targeted" modification of the structure functions of the assimilation within an area of large sensitivity to the initial conditions of cyclogenesis events is shown to have a clear positive impact in this series of FASTEX cases. This procedure can be efficient for applications, such as targeting or meteorological situations, characterized by a strong, large-scale flow where the numerical forecast can fail at short-range.

The gain of this method comes from the fact that it modifies the background forecast covariance matrix B. By doing so, it gives a better approximation of it locally, i.e. it ties it to the actual flow. The current $\mathbf{B}$ used in operational centres is static. This is known to be a disadvantage in cases of strong, rapidly evolving systems. To put a flow dependent constraint on the background part of the assimilation is an ongoing research area (which has become a reality in the operational centres that use 4D-Var assimilation) with, for example, low rank Kalman filters (Fisher, 1998), or Houtekamer and Mitchell (1998) for an estimate of the forecast errors statistics with an ensemble Kalman filter. The interest in the method described here is that it is not too costly as far as only one direction of the unstable subspace is considered.

The limits of the method now have to be explored. It will be necessary to characterize flow regimes for which one direction is a sufficient approximation of the unstable subspace. It will also be required to compare the benefit of this simple strategy with a "targeted" simplified Kalman filter, i.e. "targeted" in that the unstable subspace should be com- 
puted on a specific weather system. Next, one has to consider going to a 4D-Var framework. This may or may not provide the same benefits since 4D-Var is able to develop implicitly flow-dependent structure functions (Thépaut et al., 1996).

Another aspect to investigate is the appropriate value for the parameter $\sigma_{1}$. For the time being, the assumption is to give no confidence to the background field in the sensitive direction. It could be of interest to test a method, such as the adaptive tuning of the assimilation parameters (Desroziers and Ivanov, 2000), in order to determine an optimal value of $\sigma_{1}$ depending on the meteorological situation.

\section{Appendix A Householder matrices}

Householder matrices are square and orthogonal matrices. They have a simple form:

$\mathbf{X}=\mathbf{I}_{n}-2 \frac{\boldsymbol{v} \boldsymbol{v}^{T}}{\left\|\boldsymbol{v}^{2}\right\|}$,

where $\mathbf{I}_{n}$ is the identity matrix, $\boldsymbol{v}$ is a vector:

$\boldsymbol{v}=\boldsymbol{a}-\boldsymbol{r}, \quad \boldsymbol{a}=\left(\begin{array}{c}a_{1} \\ \vdots \\ \vdots \\ a_{n}\end{array}\right), \quad \boldsymbol{r}=\left(\begin{array}{c}\|a\| \\ 0 \\ \vdots \\ 0\end{array}\right)$.

Then it follows:

$\mathbf{X} \boldsymbol{a}=\boldsymbol{r}$.

Acknowledgements. The authors want to thank M. Fisher for useful ideas at the beginning of this work and help during the experiments. They also thank P. Arbogast and G. Desroziers for discussions and constructive comments on this work. The latter is especially thanked for important help on Sect. 2 and Sect. 3. We acknowledge A. Joly for constructive comments and help on this manuscript. Three anonymous reviewers who provided helpful comments on an earlier version of this manuscript are acknowledged.

\section{References}

Bergot, T.: Adaptive observations during FASTEX: A systematic survey of upstream flights, Quart. J. Roy. Meteor. Soc, 125, 3271-3298, 1999.

Bergot, T.: Influence of the assimilation scheme on the efficiency of adaptive observations, Quart. J. Roy. Meteor. Soc, 127, 635-661, 2001.

Bishop, C. H. and Toth, Z.: Ensemble transformation and adaptive observations, J. Atmos. Sci.,56, 1748-1765, 1999.

Bouttier, F., Derber, J., and Fisher, M.: The 1997 revision of the $J_{b}$ term in 3D/4D-Var, ECMWF Tech. Memo. 238 (available from ECMWF, Reading, UK), 1997.

Cammas, J-P., Pouponneau, B., Desroziers, G., Santurette, P., Joly, A., Arbogast, P., Mallet, I., Caniaux, G., and Mascart, P.: FASTEX IOP17 cyclone: Introductory synoptic study with field data. Quart. J. Roy. Meteor. Soc, 125, 3393-3414, 1999.
Courtier, P., Freydier, C., Geleyn, J-F., Rabier, F., and Rochas, M.: The Arpege project at Météo-France, In: Proceedings of the ECMWF workshop on numerical methods in atmospheric models, (Eds) ECMWF, Reading, UK, 9-13, 1991.

Courtier, P., Andersson, E., Heckley, W., Pailleux, J., Vasiljević, D., Hamrud, M., Hollingsworth, A., Rabier, F., and Fisher, M.: The ECMWF implementation of three-dimensional variational assimilation (3D-Var), I: Formulation, Quart. J. Roy. Meteor. Soc, 124, 1783-1807, 1998.

Desroziers G. and Ivanov, S.: Diagnosis and adaptive tuning of information error parameters in a variational assimilation, Quart. J. Roy. Meteor. Soc., 127. 1433-1452, 2001.

Desroziers, G., Pouponneau, B., Thépaut, J.-N., Janisková, M., and Veersé, F.: Four-dimensional variational analyses of FASTEX situations using special observations Quart. J. Roy. Meteor. Soc, 125, 3339-3358, 1999.

Emanuel, K., Raymond, D., Betts, A., Bosart, L., Bretherton, C., Droegemeir, K., Farrell, B., Fritsch, J. M., Houze, R., Le Mone, M., Lilly, D., Rotunno, R., Shapiro, M., Smith, R., and Thorpe, A.: Report of the first prospectus development team of the U.S. weather research program to NOAA and the NSF Bull. Am. Meteorol. Soc., 76, 1194-1208, 1995.

Fisher, M.: Developement of a simplified Kalman filter, ECMWF Tech. Memo. 260 (available from ECMWF, Reading, UK), 1998.

Hello, G., Lalaurette, F., and Thépaut, J.-N.: Combined used of sensitivity information and observations to improve meteorological forecasts: A feasibility study applied to the "Christmas storm" case, Quart. J. Roy. Meteor. Soc, 126, 621-647, 2000.

Houtekamer, P. L. and Mitchell, H. L.: Data assimilation using an ensemble kalman filter technique, Mon. Wea. Rev., 126, 796$811,1998$.

Joly, A., Browning, K. A., Bessemoulin, P., Cammas, J. P., Caniaux, G., Chalon, J. P., Clough, S. A., Dirks, R., Emanuel, K. A., Eymard, L., Lalaurette, F., Gall, R., Hewson, T. D., Hildebrand, P. H., Jorgensen, D., Langland, R. H., Lemaitre, Y., Mascart, P., Moore, J. A., Persson, P. O. G., Roux, F., Shapiro, M. A., Snyder, C., Toth, Z., and Wakimoto, R. M.: Overview of the field phase of the Fronts and Atlantic Storm-Track Experiment (FASTEX) project, Quart. J. Roy. Meteor. Soc, 125, 3131-3163, 1999.

Rabier F., Klinker, E., Courtier, P., and Hollingsworth, A.: Sensitivity of forecast errors to initial conditions, Quart. J. Roy. Meteor. Soc, 122, 121-150, 1996.

Snyder, C.: Summary of an informal workshop on adaptive observations and FASTEX, Bull. Am. Meteorol. Soc., 77, 953-965, 1996.

Szunyogh, I., Thoth, Z., Emanuel, K. A., Bishop, C. H., Snyder, C., Morss, R. E., Woolen, J., and Marchok, T.: Ensemble-based targeting experiments during FASTEX: The effect of dropsonde data from the Lear jet, Quart. J. Roy. Meteor. Soc, 125, 31893217, 1999.

Thépaut, J.-N., Alary, P., Caille, P., Cassé, V., Geleyn, J.-F., Moll, P., Pailleux, J., Piriou, J.-M., Puech, D., and Taillefer, F.: The operational global data assimilation system at météo-France. in: Proceedings of HIRLAM 4 workshop on Variational Analysis in Limited Area Models, (Eds) HIRLAM 4 project, c/o Met Eireann, Glasnevin Hill, Dublin 9, Ireland, 25-31, 1998.

Thépaut, J.-N., Courtier, P., Belaud, G., and Lemaître, G.: Dynamical structure functions in a four-dimensional variational assimilation, A case study, Quart. J. Roy. Meteor. Soc, 122, 535-561, 1996. 